\title{
Location errors in partial-report bar-probe experiments: In search of the origin of cue-alignment problems
}

\author{
RUCHAMA HAGENAAR and A. H. C. VAN DER HEIJDEN \\ Leiden University, Leiden, The Netherlands
}

\begin{abstract}
In studies using Averbach and Coriell's (1961) partial-report bar-probe paradigm with linear arrays, most errors involve the naming of an item that was in the display but in a position other than the cued one. Up to now, there is no general agreement on the origin of these location errors. Point of departure in this paper is that part of the location errors arises from inappropriate application of the cue. It is tested whether this originates from problems to perceive the position of the cue ("cuedisplacement hypothesis") or from confusion about the order of the items in the array ("item-order hypothesis"). The results of two bar-probe experiments are reported. A novel, crucial, finding in both experiments is that, among the location errors, there was a preponderance of response letters that came from the central side of the cued item. In the second experiment, this was observed not only in the usual postcue conditions but also when the cue preceded the array. These results positively corroborate the cue-displacement hypothesis and do not support the item-order hypothesis: The cue tends to be perceived more toward the center of the visual field than it actually is exposed-that is, there is a central drift of the cue.
\end{abstract}

The partial-report technique is a powerful tool in the study of selective attention and memory processes in vision. In this technique, subjects are briefly exposed to a configuration of letters and a cue that specifies which part of the display is to be reported. In the variety introduced by Averbach and Coriell (1961)-the partialreport bar-probe paradigm - one or several rows of letters are displayed, and only one letter has to be named. A visual cue (e.g., a bar or an arrow) is used to indicate the target letter. The cue is presented just before, during, or at various intervals after exposure of the letter display.

From these partial-report bar-probe studies, a consistent pattern of results has emerged (see, e.g., Averbach \& Coriell, 1961; Hagenaar \& van der Heijden, 1995; Mewhort, Campbell, Marchetti, \& Campbell, 1981; Mewhort, Marchetti, \& Campbell, 1982; Townsend, 1973). The first robust finding is that accuracy of report depends on the moment of appearance of the cue. If the cue just precedes the letter display or is shown simultaneously, accuracy is quite high (approximately $75 \%$ correct). If the cue follows the display immediately, accuracy of report is still high. Accuracy decreases gradually when the cue is further delayed. At a delay of about $250 \mathrm{msec}$ after display termination, accuracy reaches an asymptotic level of about $35 \%$ correct. The second robust finding is that accuracy is higher for letters displayed at the center and the ends of the row(s) than for letters shown between the center and

Correspondence should be addressed to A. H. C. van der Heijden, Unit of Experimental and Theoretical Psychology, Leiden University, P.O. Box 9555, 2300 RB Leiden, The Netherlands (e-mail: heijden@ rulfsw.leidenuniv.nl). end positions: The percentage correct reports are distributed in the shape of a "W" across positions.

Initially, the less than perfect performance in this task was attributed to difficulty in identifying the target letter properly (see, e.g., Averbach \& Coriell, 1961; see also Estes, 1978). Later, a detailed analysis of the errors was introduced. In this analysis, each erroneous response was categorized as either an item error or a location error. An item error refers to report of a letter that was not in the array; a location error involves report of a letter that was in the array but not in the cued position. The outcome of studies using this error analysis was surprising: The great majority of errors appeared to be location errors, whereas the number of item errors was very modest (see, e.g., Hagenaar \& van der Heijden, 1995; Hagenzieker, van der Heijden, \& Hagenaar, 1990; Mewhort et al., 1981; Mewhort et al., 1982; Townsend, 1973). Moreover, the location errors nearly mirrored the pattern of correct reports: A strong increase with cue delay and an M-shaped serial position curve were found. In contrast, item errors showed only a slight rise as a function of cue delay and a weak $U$-shape across positions.

Studies in which the observed location errors were further analyzed revealed that most location errors consisted of report of a letter that was in a position adjacent to the target (i.e., near-location errors). Only on a minority of trials did subjects report a letter that was further removed from the target (i.e., far-location errors; see, e.g., Hagenaar \& van der Heijden, 1995; Hagenzieker et al., 1990; Mewhort et al., 1981).

From these findings, it was concluded that locating difficulties are a major source of errors in partial-report bar-probe experiments (see, e.g., Lowe, 1975; Townsend, 
1973). Mewhort and colleagues associated the limitations in locating both with difficulty retaining a stable memory representation of the positions of the letters in the string and with difficulty matching cue position and target letter position (see, e.g., Campbell \& Mewhort, 1980; Mewhort, 1987; Mewhort, Butler, Feldman-Stewart, \& Tramer, 1988; Mewhort et al., 1981; Mewhort et al., 1982). In a simulation study, Mewhort et al. (1988) demonstrated that, with short cue delays, problems with matching cue position and target position form the main cause of location errors in bar-probe tasks.

Location errors due to cue-alignment problems are the topic of this paper. For the purpose of the present study, we define cue-alignment problems as limitations in the process of relating the position of the cue to that of the target because of spatial drift of the cue, of the target, or of both. (Please note that this definition differs from the definitions presented by Mewhort, 1987, Mewhort et al., 1988, and van der Heijden, 1992, which focus only on the spatial position of the cue.) What we basically want to know is how such cue-alignment problems may affect partial-report bar-probe performance. Shedding some light on this issue is not just of local interest. Because of the close relationship between position and selective attention (see, e.g., Eriksen, 1990; van der Heijden, 1992, 1993), gaining insight into the origin of the cue-alignment problems also provides further knowledge about the functioning of selective attention.

Unfortunately, the partial-report bar-probe literature provides no information for generating detailed hypotheses about what may lie at the basis of cue-alignment problems in partial-report bar-probe experiments. There are, however, two pieces of extraneous empirical evidence, both hinting at peculiar problems with position perception, that can be used for generating hypotheses. The first piece was provided by Estes, Allmeyer, and Reder (1976) and can be taken to suggest that the cue-alignment problems are caused by specific problems with perception of the positions of the letters in the arrays. The second piece of evidence was reported by Rauk and Luuk (1980) and can be taken to suggest that systematic erroneous perception of the position of the cue in visual space underlies the cue-alignment problems.

Estes et al. (1976) investigated the properties of the serial position functions that were found for tachistoscopic whole report. They observed that, with horizontal four-letter rows as stimuli, subjects had great difficulty in correctly ordering the letters to be reported; the subjects made many "transposition errors" (for similar findings see, e.g., Krumhansl, 1977, and Krumhansl \& Thomas, 1976, 1977). In the present context, one further outcome of this investigation is of importance. The "transposition errors exhibit a pronounced peripheral-to-central drift." This observation was taken to reflect a tendency of the letters "to move centrally, whether the transposed item displaces another letter or occupies the position of a letter which was not reported from the given display" (Estes et al., 1976, p. 11).

Rauk and Luuk (1980) investigated the accuracy of the absolute judgment of the spatial position of an iso- lated dot that could be regarded as a member of a horizontal (or vertical) one-dimensional dot pattern. They observed that subjects had great difficulty naming the position of such a single dot (for similar findings, see, e.g., Harcum, 1964, 1970). In the present context, one further outcome of this research is of significance. It appeared that "in most cases the means of the erroneous responses were shifted towards the central fixation point." This observation was taken to reflect the existence of "a general tendency to estimate the position of an object as if it was more centrally located than it actually was" (Rauk \& Luuk, 1980, p. 150).

\section{EXPERIMENT 1}

Estes et al.'s (1976) observation that letters in a string tend to be seen as displaced in the direction of the central fixation point and Rauk and Luuk's (1980) observation that an isolated dot tends to undergo the same fate enable us to formulate two major hypotheses about the cause of cue-alignment problems in partial-report barprobe tasks that can be easily tested by means of a further analysis of the location errors.

The first hypothesis assumes that the letters in the array tend to be perceived as having moved toward the central point of fixation as reported by Estes et al. (1976), but that, contrary to Rauk and Luuk's (1980) observations, no problems with the perception of the spatial position of the cue occur. If this is what happens, errors resulting from cue-alignment problems must consist mainly of the names of letters that occupied peripheral positions relative to the position of the cue. In other words, the inward movement of the letters produces an abundance of "peripheral" location errors.

The second hypothesis assumes that there is a tendency to perceive the cue as having moved in the direction of the point of fixation, such as the dot in Rauk and Luuk's (1980) experiments, but that, contrary to Estes et al.'s (1976) observations, no perceptual problems with the position of the letters in the array occur. If this is what happens, errors resulting from cue-alignment problems must consist mainly of the names of letters that were exposed in central positions relative to the position of the cue. In other words, the inward movement of the cue produces an abundance of "central" location errors.

To investigate which of these two main hypotheses is most in accord with the data, a partial-report bar-probe experiment was carried out. For each location error, it was established whether, relative to the position of the target, the erroneous response letter came from a position toward the center or toward the periphery of the array. Because in conventional partial-report bar-probe experiments many different factors contribute to the outcome (see, e.g., Hagenaar \& van der Heijden, 1995; Mewhort, 1987; Mewhort et al., 1988; van der Heijden, 1992), we used the simplified version introduced by Mewhort and Campbell (1978). In this version, instead of variable letter arrays, one invariant digit array (1234567) is used as the stimulus. From our previous research, we know that 
this task gives a reliable look at the cue-alignment processes involved in the conventional bar-probe paradigm (see Hagenzieker et al., 1990; van der Heijden, 1992).

\section{Method}

Subjects. The subjects were 20 paid volunteers, recruited from the student population at the University of Leiden. All of them reported normal or corrected-to-normal vision.

Apparatus. The stimuli were shown on a fast display screen (Vector General Graphics Display) equipped with fast-decaying P4-phosphor. Screen size was reduced to a rectangle of about $8.0^{\circ}$ $\times 9.5^{\circ}$ of visual angle by means of a window cut in a sheet of black paper. The subject was seated at a table in front of the screen at a viewing distance of about $85 \mathrm{~cm}$. The subject initiated stimulus exposure by pressing a button on the table surface in front of him/her. The presentation of trial events and the recording of responses were controlled by a PDP $11 / 34$ computer.

The experiment was run in a dimly illuminated room. The luminance of the display background was approximately $5.4 \mathrm{~cd} / \mathrm{m}^{2}$, and the luminance of the stimulus display was approximately $29.8 \mathrm{~cd} / \mathrm{m}^{2}$. Luminances were measured by means of a United Detector Technology optometer (Model 80X).

Stimulus material. The same linear array--consisting of the digits 1 through 7 , always in this sequence and with the digit 1 in the leftmost position--was presented to all subjects on all trials. The continuous-line "white" digits on the relatively dark screen were generated by the Vector General hardware. The digits subtended maximum visual angles of about $0.30^{\circ}$ horizontally and about $0.40^{\circ}$ vertically. The width of the arrays was about $3.10^{\circ}$, and the interitem spaces were at least $0.17^{\circ}$. The cue was an arrow above one of the digit positions. The length of the arrow was about $0.35^{\circ}$, and its maximal width was $0.24^{\circ}$. The nearest contours of arrow and cued digit were separated by approximately $0.30^{\circ}$.

Design. A randomized-block factorial design (Kirk, 1968) was employed. Target position and cue delay were the within-subject variables. Each subject saw a unique series of 245 trials, being five replications of the factorial combination of seven target positions and seven cue delays (interstimulus intervals [ISIs] of $0,50,100$, $150,200,300$, and $400 \mathrm{msec}$ ). The order of presentation was fully randomized. The experimental trials were preceded by a series of 60 practice trials.

Procedure. Each subject served in one individual 45-min session. Each session started with a 5-min period for adaptation to the reduced illumination level in the experimental room. Prior to stimulus exposure, the subject was informed about the task and the invariant seven-digit stimulus array.

On a trial, the subject had to look at a dim fixation cross shown on the empty screen and to initiate stimulus presentation when the cross was sharply in focus. The fixation cross (situated just below the middle position of the array) disappeared on array appearance. After an interval of variable length, the array was followed by an arrow pointing to one of the item positions. Finally, the fixation cross reappeared. The subject was requested to name the digit in the cued position and to guess if unsure. Stimulus exposure time was $30 \mathrm{msec}$ and cue duration was $50 \mathrm{msec}$.

The test trials were preceded by six series of 10 practice trials. Stimulus duration in these series ranged from $400 \mathrm{msec}$ (first series) to $30 \mathrm{msec}$ (last series). Feedback was given on practice trials only.

\section{Results}

For each subject, the erroneous responses were scored as either near-location errors or far-location errors, depending on whether the response involved a digit adjacent to the target (near) or a digit farther removed (far).
The overall average proportions were .222 for nearlocation errors and .005 for far-location errors. Figure la shows the proportions of errors as a function of cue delay, averaged over target position. Figure $1 \mathrm{~b}$ shows the proportions as a function of target position, averaged over cue delay.

The near-location errors were submitted to further analysis, but the far-location errors were not since they were too rare to make analysis meaningful. An analysis of variance (ANOVA) showed that the effect of cue delay was highly significant $\left[F(6,114)=17.997, M S_{\mathrm{e}}=0.037\right.$, $p<.001]$. Duncan's test for multiple comparisons showed that the proportions of near-location errors for the delays of 0 and $50 \mathrm{msec}$ differed significantly from each other and from any other proportion and that the proportion for the delay of $100 \mathrm{msec}$ was significantly lower than those corresponding to the delays of 300 and $400 \mathrm{msec}$, $\left(M S_{\mathrm{e}}=0.037, d f=114, p<.05\right)$.

The effect of target position was also highly significant $\left[F(6,114)=11.923, M S_{\mathrm{e}}=0.005, p<.001\right]$. A trend analysis confirmed the quartic trend in the M-shaped curve $\left[F(1,114)=37.144, M S_{\mathrm{e}}=0.005, p<.001\right]$. The interaction of cue delay and target position was not significant.

Table 1 includes the information on the spatial direction of the location errors, and it gives, in proportions, the frequencies with which each position supplied the response digit for a particular target position.

Table 1 clearly shows that the near-location errors were strongly biased toward the center of the array. Obviously, for the end positions (Positions 1 and 7), nearlocation errors could occur only on the foveal side. Ignoring these end positions, and also the middle position (Position 4), which cannot generate central location errors, we found for Positions 2, 3, 5, and 6 an average proportion of central near-location errors of .922 and of peripheral near-location errors of .078 .

\section{Discussion}

The results of Experiment 1 are consistent with and fully replicate the results of experiments with similar stimuli reported by Mewhort and Campbell (1978) and by Hagenzieker et al. (1990). On about a quarter of the trials, the subjects failed to name correctly the target item in a predictable linear digit array. Nearly all errors were near-location errors; the number of far-location errors was negligible. The frequency of near-location errors appeared to depend on the position of the cue and on the moment of its presentation: The errors were distributed in an M-shape over array positions; only a few nearlocation errors occurred at an ISI of $0 \mathrm{msec}$, and their number gradually increased with delay of the cue.

The theoretically interesting outcome is a phenomenon not reported before: The overwhelming majority (over $90 \%$ ) of the near-location errors consisted of the name of a digit on the foveal side of the indicated digit (i.e., were central near-location errors). Less than $10 \%$ of the nearlocation errors were peripheral ones. This novel finding is of theoretical importance because, as elaborated in the 

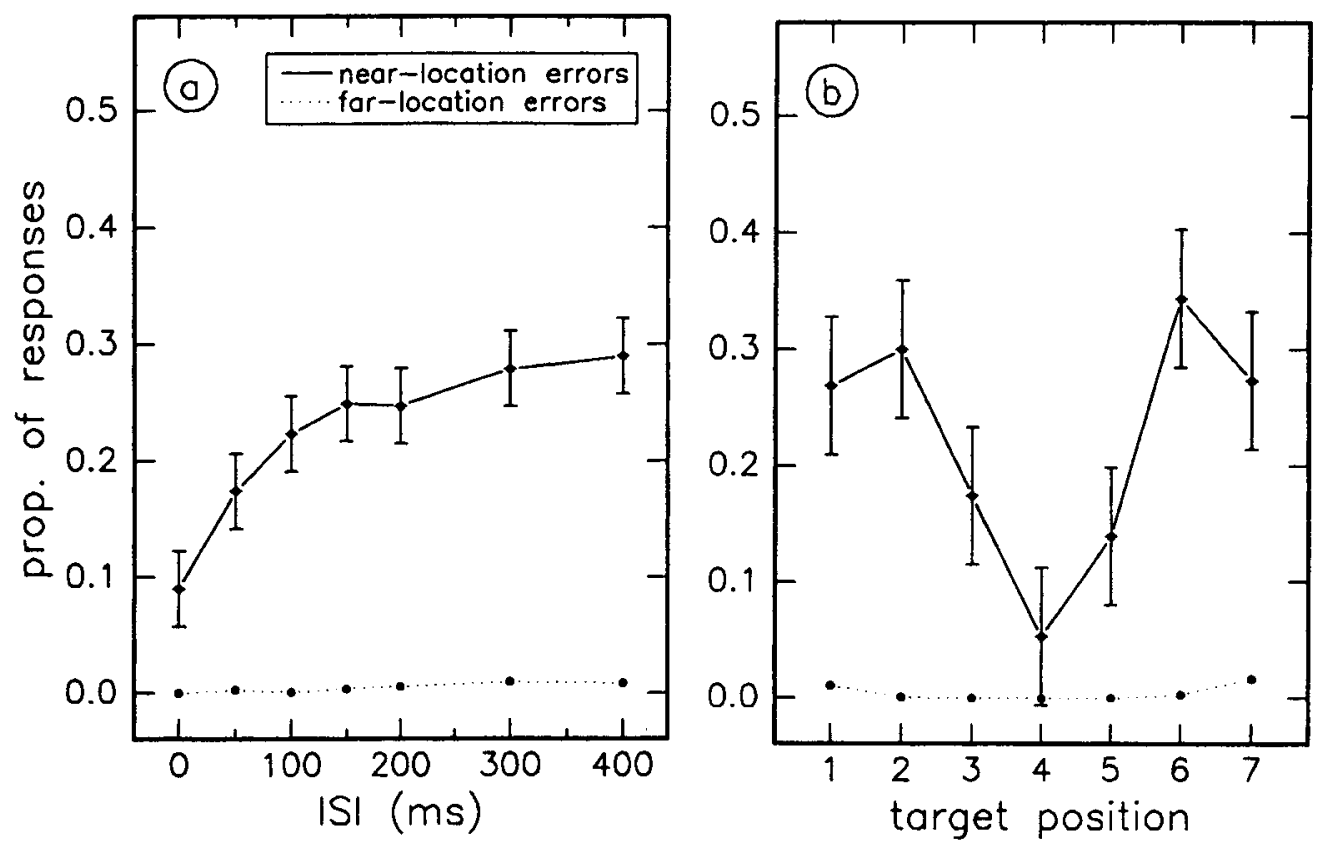

Figure 1. Frequencies of error responses as a function of cue delay (a) and of target position (b) in Experiment 1. (The alpha level for the confidence intervals is at $5 \%$.)

introduction of this paper, it strongly suggests that the location errors occurring with the present type of arrays were caused by cue-alignment problems resulting from apparent displacement of the cue toward the central point of fixation, as suggested by Rauk and Luuk's (1980) studies. The corresponding virtual absence of peripheral near-location errors does not provide any support for the alternative hypothesis stating that location errors are caused by cue-alignment problems resulting from apparent displacement of the items toward the center of the visual field, as was suggested by Estes et al.'s (1976) study.

Admittedly, this conclusion may be true only for a barprobe task with unconventional invariant linear digit arrays, as used in the present experiment. While the pattern of location errors is strikingly similar to the one that is generally obtained in conventional bar-probe experiments with variable letter arrays - an abundance of nearlocation errors, an increase of near-location errors with increasing cue-delays, and an M-shaped distribution of location errors over array positions-there are at least two reasons to doubt whether, in the conventional task, the majority of near-location errors can be central ones.

The first, rather trivial, reason is that, in conventional bar-probe experiments, many sources of errors contribute to the total pattern of results (see, e.g., Hagenaar \& van der Heijden, 1995; Mewhort, 1987; Mewhort et al., 1988). In such experiments, therefore, evidence for central near-location errors resulting from apparent movement of the cue may be obscured by errors induced by other factors.

The second, more fundamental, reason is that some evidence is available suggesting that peripheral nearlocation errors resulting from apparent movement of items toward the center of the visual field can be obtained only with variable - hence unpredictable-linear (letter) arrays (see, e.g., Estes et al., 1976, p. 10 and p. 13). In other words, the invariant-digit bar-probe paradigm might be biased in the sense that it possibly precludes the occurrence of peripheral near-location errors.

Table 1

Positions That Supplied the Response Digit per Target Position

\begin{tabular}{cccccccc}
\hline \multirow{2}{*}{$\begin{array}{c}\text { Position of } \\
\text { Target Digit }\end{array}$} & 1 & 2 & 3 & 4 & 5 & 6 & 7 \\
\hline \cline { 2 - 7 }$y$ & .720 & .269 & .011 & .000 & .000 & .000 & .000 \\
2 & .011 & .699 & .289 & .001 & .000 & .000 & .000 \\
3 & .000 & .007 & .826 & .167 & .000 & .000 & .000 \\
4 & .000 & .000 & .026 & .947 & .027 & .000 & .000 \\
5 & .000 & .000 & .000 & .114 & .861 & .024 & .000 \\
6 & .000 & .000 & .000 & .003 & .324 & .653 & .020 \\
7 & .000 & .000 & .000 & .000 & .017 & .273 & .710 \\
\hline
\end{tabular}

Note-Underlined diagonal values denote the proportions of correct reports; offdiagonal values denote the proportions of location errors. Italicized values represent near-location errors. 
To investigate the generality of the phenomenon of the central near-location errors, we analyzed the distribution of location errors obtained in two variable-letter bar-probe experiments. Table 2 presents the results reported by Hagenaar and van der Heijden (1995). Like Table 1, it shows, for each cued location, which locations provided the response letters. The table convincingly shows that in this letter experiment, just as in the present digit experiment, near-location errors tend to be central ones $(.744$ central and .256 peripheral near-location errors for Target Positions 2, 3, 5, and 6). Table 3 presents, in a similar form, the results of an eight-letter-array bar-probe experiment reported by Mewhort et al. (1981, Condition 1, zero-order and minus-two-order approximations to English). Again, for Target Positions 2, 3, 6, and 7 (the end positions and two middle positions ignored), near-location errors tend to be centrally directed (.652 central and .348 peripheral errors), despite the deviating pattern for Target Position 2.

The outcome of these two post hoc analyses strongly indicates that, in conventional bar-probe experiments, near-location errors are predominantly central ones. This, in turn, strongly suggests that, in the conventional letter bar-probe paradigm, the apparent movement of the cue in visual space toward the center of the visual field is the major cause of the cue-alignment problems. The aim of Experiment 2 was to further investigate this hypothesis.

\section{EXPERIMENT 2}

The new phenomenon observed in Experiment 1 is that, in a bar-probe experiment with a predictable digit array, near-location errors consist mainly of the names of digits that were shown on the central side of the cued position. As elaborated in the introduction to Experiment 1 , this outcome can be taken to indicate that cuealignment problems arise from apparent movements of the cue in visual space in the direction of the fixation point, such as the dot in Rauk and Luuk's (1980) experiments. This, in turn, suggests that, in conventional barprobe studies, with variable letter sequences as stimuli, the systematic misperception of the position of the cue in visual space might be the main cause of the near-location errors. This interpretation is, however, not the common one. Most researchers emphasize one or another kind of item-order problem as the major cause (see, e.g, Campbell \& Mewhort, 1980; Mewhort et al., 1988). So, while the reanalyses of Hagenaar and van der Heijden's (1995) and Mewhort et al.'s (1981) data already supported this new position, a further test of the "cue-displacement hypothesis" is in order.

An appropriate way of assessing the viability of the hypothesis that "movements" of the cue in visual space in the direction of the fixation point are at the basis of the near-location errors in conventional letter bar-probe studies is to compare performance under conditions of postexposure of the cue with performance under conditions of preexposure of the cue. There is no reason to assume that it is easier or more difficult to perceive the absolute position of a cue when it precedes the display than when it follows the display. So, apparent movements of the cue toward the point of fixation have to be expected with postexposure of the cue as well as with preexposure of the

Table 2

Positions of Response Letters in Partial-Report Bar-Probe Experiments With Variable Linear Letter Arrays Reported by Hagenaar and van der Heijden (1995)

\begin{tabular}{|c|c|c|c|c|c|c|c|c|}
\hline \multirow{2}{*}{$\begin{array}{l}\text { Position of } \\
\text { Target Letter }\end{array}$} & \multicolumn{7}{|c|}{ Position of Response Letter } & \multirow{2}{*}{$\begin{array}{l}\text { Letter No } \\
\text { in Array }\end{array}$} \\
\hline & 1 & 2 & 3 & 4 & 5 & 6 & 7 & \\
\hline 1 & .524 & .098 & .074 & .012 & .019 & .014 & .012 & .248 \\
\hline 2 & $.1 / 9$ & .186 & .338 & .069 & .012 & .029 & .012 & .236 \\
\hline 3 & .036 & .050 & .571 & .167 & .024 & .014 & .012 & .126 \\
\hline 4 & .002 & .005 & .026 & .895 & .036 & .007 & .002 & .026 \\
\hline 5 & .005 & .005 & .017 & .102 & .760 & .038 & .007 & .067 \\
\hline 6 & .019 & .019 & .029 & .038 & 300 & .295 & .107 & .193 \\
\hline 7 & .012 & .029 & .019 & .012 & .064 & .124 & .495 & .245 \\
\hline
\end{tabular}

Note--Underlined values represent proportions of correct reports. Italicized values represent proportions of near-location errors.

Table 3

Positions of Response Letters in Partial-Report Bar-Probe Experiments With Variable Linear Letter Arrays Reported by Mewhort et al. (1981, Condition 1)

\begin{tabular}{cccccccccc}
\hline $\begin{array}{c}\text { Position of } \\
\text { Target Letter }\end{array}$ & 1 & 2 & 3 & 4 & 5 & 6 & 7 & 8 & $\begin{array}{c}\text { Letter Not } \\
\text { in Array }\end{array}$ \\
\cline { 2 - 9 } & .568 & .036 & .039 & .029 & .039 & .011 & .011 & .021 & .246 \\
2 & .232 & .104 & .161 & .061 & .061 & .014 & .007 & .021 & .339 \\
3 & .054 & .043 & .368 & .186 & .050 & .014 & .011 & .011 & .264 \\
4 & .025 & .011 & .046 & .736 & .029 & .021 & .021 & .011 & .100 \\
5 & .018 & .000 & .014 & .057 & .729 & .021 & .011 & .004 & .146 \\
6 & .007 & .004 & .007 & .029 & .229 & .457 & .071 & .029 & .168 \\
7 & .014 & .018 & .021 & .018 & .114 & .232 & .204 & .139 & .239 \\
8 & .007 & .032 & .032 & .004 & .029 & .107 & .057 & .379 & .354 \\
\hline
\end{tabular}

Note-Underlined values represent proportions of correct reports. Italicized values represent proportions of near-location errors. 

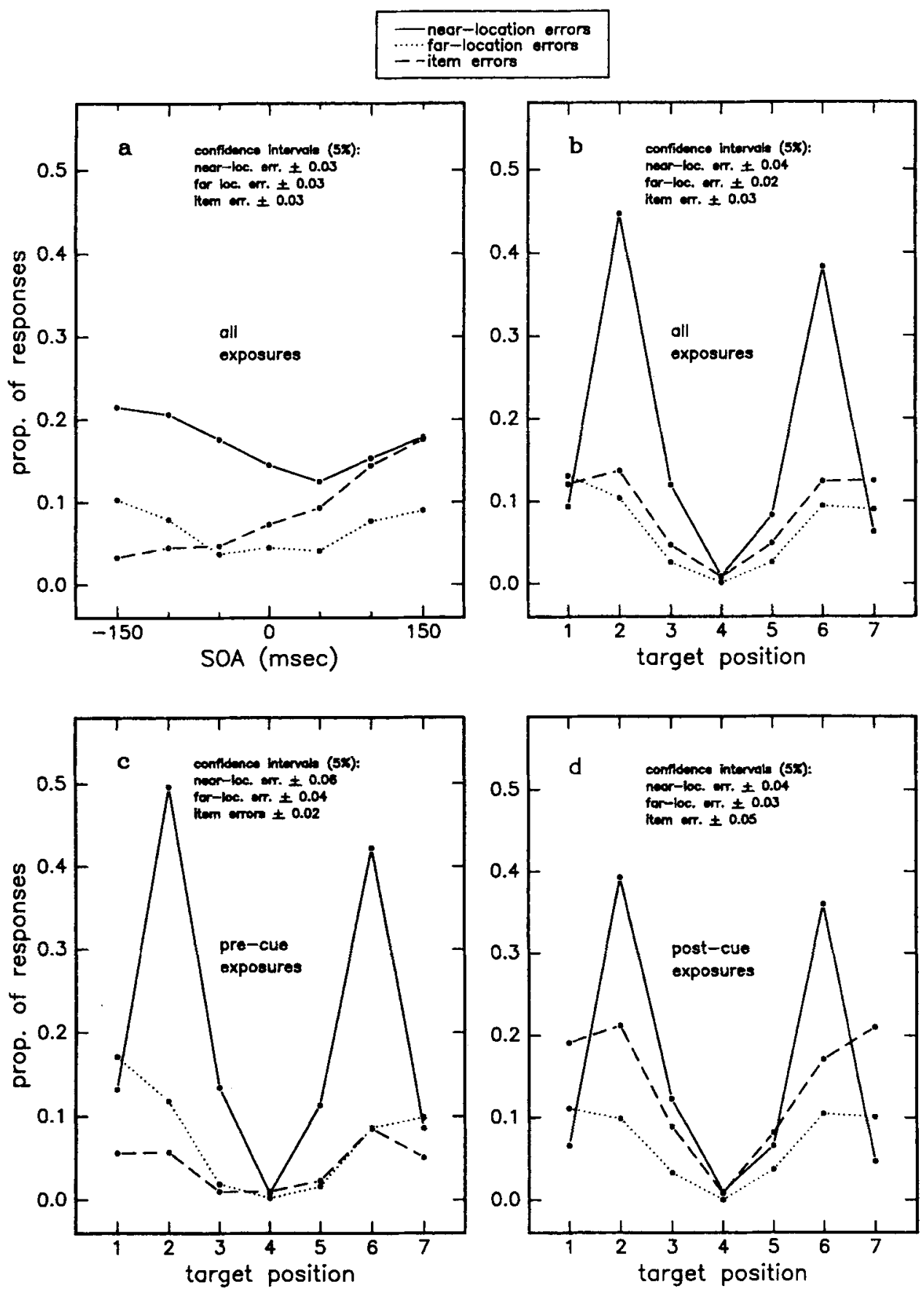

Figure 2. Frequencies of error responses in Experiment 2 as a function of cue delay for all conditions (a), of target position for all conditions (b), and of target position separately for precue conditions (c) and for postcue conditions (d).

cue. Therefore, if the cue-displacement hypothesis is true, and near-location errors arise mainly from cue mislocation in visual space, (1) near-location errors will occur both with pre- and postexposure of the cue and, (2) under both conditions, the near-location errors will be mainly central ones.

It is of importance to note that these predictions are unique for the cue-displacement hypothesis. The itemorder hypothesis predicts that near-location errors will be mainly peripheral near-location errors (see the introduction to Experiment 1). Moreover, from this hypothesis follows that item-order problems can arise only after exposure of the array. Therefore, according to this hypothesis, near-location errors must be expected mainly in postexposure conditions and not with preexposure of the cue.

To assess the viability of the cue-displacement hypothesis, we performed a bar-probe experiment with conventional variable letter stimuli and varied the moment of 
cue presentation from $150 \mathrm{msec}$ before to $150 \mathrm{msec}$ after array presentation. This experiment tests whether, as predicted by the cue-displacement hypothesis, in conventional letter bar-probe experiments, (1) with postexposure of the cue, near-location errors are indeed mainly central near-location errors, as suggested by our Experiment 1 and our reanalysis of Hagenaar and van der Heijden's (1995) and Mewhort et al.'s (1981) data, and (2) with preexposure of the cue, central near-location errors are also observed.

\section{Method}

Subjects. Nine students at the University of Leiden served as paid subjects. All of them reported normal or corrected-to-normal vision. None of them participated in Experiment 1.

Apparatus. The equipment for presentation of the stimuli and recording of the responses was identical to that used in Experiment 1 .

Stimulus material. The stimuli were variable, linear letter arrays of the same dimensions as the digit arrays in Experiment 1. For each string, seven different letters were randomly selected from the 26 letters of the alphabet. Any letter in an array could serve as the target letter.

Design. A randomized-block factorial design was employed, with target position, cue delay, and experimental day as withinsubject variables. Each subject saw three unique series of 294 trials, being six replications of the factorial combination of seven target positions and seven cue delays (SOAs of $-150,-100,-50,0$ $+50,+100$, and $+150 \mathrm{msec}$ ). The order of presentation was fully randomized.

Procedure. Each subject served in four individual 45-min sessions: One session for practice and three for the main experiment. The sessions were separated by about $24 \mathrm{~h}$.

In the practice session, the subject was familiarized with the task with two types of stimuli. First, the subject was given practice with invariant digit arrays, as were used in Experiment 1; by doing so, the subject was enabled to get used to finding the position of the target item without being bothered by identification problems. Thereupon, the subject was presented with variable letter arrays. Of both types of arrays, the subject saw three brief series of 10 trials with decreasing exposure durations $(200,100$, and $50 \mathrm{msec}$, respectively $)$ and another three series of $49(=7$ cue delays $\times 7$ target positions) trials with the same exposure time as in the experimental sessions. In these practice trials, the subjects got detailed feedback on their responses.

In each of the three experimental sessions with variable letter arrays, the subject received a unique series of 294 trials (see Design section). The subjects were instructed to name the item indicated by the arrow. Stimulus duration was $50 \mathrm{msec}$, as was cue duration. Each test series was preceded by 40 practice trials showing invariant digit arrays and 30 trials showing variant letter arrays.

\section{Results}

Each erroneous response was scored as a near-location error (the response letter was a letter adjacent to the target), a far-location error (the response letter was in the display but farther removed from the target letter), or an item error (the response letter was not in the array at all).

The overall proportions were .171 for near-location errors, .067 for far-location errors, and .087 for item errors. Figures $2 \mathrm{a}$ and $2 \mathrm{~b}$ show the proportions of the three error categories as a function of cue delay and of target position, respectively; Figures $2 \mathrm{c}$ and $2 \mathrm{~d}$ display the pro- portions of errors as a function of target position separately for the precue condition and the postcue condition.

The three types of errors were subjected to separate ANOVAs. In none of these analyses was any effect of days. The other variables produced the following effects.

Near-location errors. The effect of cue delay was highly significant $\left[F(6,48)=6.404, M S_{\mathrm{e}}=0.031, p<.001\right]$. A trend analysis confirmed the quadratic trend in the data $\left[F(1,48)=17.297, M S_{e}=0.031, p<.01\right]$. The effect of target position was highly significant $[F(6,48)=60.369$, $\left.M S_{\mathrm{e}}=0.092, p<.001\right]$. No interaction of cue delay $\times$ target position was found.

Far-location errors. The effect of cue delay was significant $\left[F(6,48)=3.630, M S_{\mathrm{e}}=0.036, p<.025\right]$. A trend analysis confirmed the quadratic trend in the data $\left[F(1,48)=19.077, M S_{\mathrm{e}}=0.036, p<.01\right]$. The effect of target position was highly significant $[F(6,48)=20.279$, $\left.M S_{\mathrm{e}}=0.023, p<.001\right]$, and the interaction of cue delay and target position was significant $[F(36,288)=1.793$, $\left.M S_{\mathrm{c}}=0.001, p<.025\right]$

Item errors. The effect of cue delay was highly significant $\left[F(6,48)=16.025, M S_{\mathrm{e}}=0.035, p<.001\right]$, as were the effect of target position $\left[F(6,48)=16.932, M S_{\mathrm{e}}=\right.$ $0.029, p<.001]$ and the interaction of cue delay and target position $\left[F(36,288)=2.911, M S_{\mathrm{e}}=0.013, p<.001\right]$.

Direction of near-location errors. Table 4 provides detailed information on the direction of location errors. For all cue delays, a preponderance of central near-location errors was found. For Positions 2, 3, 5, and 6 (i.e., for the positions that can generate both central and peripheral near-location errors), the average proportions of central and peripheral near-location errors were .857 and .143 for the three precue conditions and .768 and .232 for the three postcue conditions.

\section{Discussion}

In most conventional bar-probe studies, only postcue conditions are used. For the present experiment, the results of the postcue conditions $(\mathrm{SOA}=50,100$, and $150 \mathrm{msec}$ ) are consistent with and replicate the results of experiments with similar stimuli as reported by, for example, Mewhort et al. (1981), Hagenzieker et al. (1990), and Hagenaar and van der Heijden (1995). The great majority of errors were location errors, whereas the number of item errors was fairly modest. Most location errors were near-location errors. Only on a minority of trials did a far-location error occur. The location errors mirrored the pattern of correct reports: A substantial increase with cue delay (of .103) and an M-shaped serial position curve were found. The item errors showed a slightly smaller increase with cue delay (of .083) and a weak U-shape across positions (see the introduction of this paper for this standard pattern of results). These data indicate that the subjects' performance in the present partial-report bar-probe task - with randomly mixed precue and postcue trials-is similar to that in the dozens of conventional bar-probe studies described in the literature. It can therefore be stated that our experiment provides 
Table 4

Positions That Supplied the Response Letters for Preexposure and

Postexposure of the Cue

\begin{tabular}{|c|c|c|c|c|c|c|c|c|}
\hline \multirow{2}{*}{$\begin{array}{l}\text { Position of } \\
\text { Target Letter }\end{array}$} & \multicolumn{7}{|c|}{ Position of Response Letter } & \multirow{2}{*}{$\begin{array}{c}\text { Letter Not } \\
\text { in Array }\end{array}$} \\
\hline & 1 & 2 & 3 & 4 & 5 & 6 & 7 & \\
\hline \multicolumn{9}{|c|}{ Preexposure } \\
\hline 1 & .642 & .132 & .097 & .043 & .014 & .014 & .002 & .056 \\
\hline 2 & .058 & 329 & .438 & .099 & .008 & .008 & .002 & .058 \\
\hline 3 & .000 & .014 & .837 & .119 & .014 & .002 & .002 & .010 \\
\hline 4 & .000 & .002 & .006 & .979 & .002 & .000 & .000 & .010 \\
\hline 5 & .002 & .004 & .006 & .088 & 848 & .025 & .004 & .023 \\
\hline 6 & .002 & .004 & .010 & .070 & .352 & .407 & .070 & .084 \\
\hline 7 & .004 & .008 & .004 & .019 & .064 & .086 & .763 & .051 \\
\hline \multicolumn{9}{|c|}{ Postexposure } \\
\hline 1 & .632 & .066 & .037 & .023 & .012 & .019 & .021 & .191 \\
\hline 2 & .070 & .296 & .323 & .060 & .014 & .008 & .016 & .212 \\
\hline 3 & .012 & .016 & .755 & .107 & .006 & .012 & .002 & .088 \\
\hline 4 & .000 & .000 & .002 & .981 & .008 & .000 & .000 & .008 \\
\hline 5 & .004 & .006 & .008 & .051 & .815 & .014 & .019 & .082 \\
\hline 6 & .021 & .014 & .021 & .049 & .243 & .364 & .117 & .171 \\
\hline 7 & .019 & .008 & .008 & .027 & .039 & .047 & .642 & .210 \\
\hline
\end{tabular}

Note-Underlined values represent proportions of correct reports. Italicized values represent proportions of near-location errors.

the appropriate data for assessing the viability of the cuedisplacement hypothesis about bar-probe performance.

With regard to the cue-displacement hypothesis, the data contain two pieces of relevant evidence. The first piece consists of the finding that, for the postcue conditions as used in most conventional bar-probe studies, the majority (.801) of the near-location errors made with targets on positions that can generate both central and peripheral near-location errors (Positions 2, 3, 5, and 6) involved naming a letter on the foveal side of the indicated letter (i.e., were central near-location errors). This outcome is in line with the findings in Experiment 1 with invariable linear digit arrays, and it also corroborates the results of our post hoc analyses of Hagenaar and van der Heijden's (1995) and Mewhort et al.'s (1981) experiments with variable letter arrays. And, as elaborated earlier, this outcome strongly indicates that the apparent displacement of the cue toward the center of the visual field is the major cause of the cue-alignment problems.

The second piece of evidence consists of a finding that, as far as we know, has never been reported. As Figures $2 \mathrm{a}$ and $2 \mathrm{~b}$ and Table 4 clearly show, in conditions with preexposure of the cue, many near-location errors were found. Moreover, Table 4 shows that most of these near-location errors were central near-location errors. (We address the function relating near-location errors to SOAs in the General Discussion section.) As indicated in the introduction of this paper, this outcome is predicted by and unique for the cue-displacement hypothesis. There is no reason to assume that it is easier or more difficult to perceive the position of the cue when it precedes the stimulus display than when it follows the stimulus display. Consequently, preexposure of the cue must produce the same pattern of results as postexposure: an abundance of near-location errors that, for the main part, are central near-location errors. Because this is exactly what we observed, the outcome with preexposure of the cue strongly supports the conclusion we have already reached on the basis of the results of Experiment 1 with predictable digit arrays, the results of the post hoc analyses of Hagenaar and van der Heijden's (1995) and Mewhort et al.'s (1981) conventional variable-letter bar-probe tasks, and the results of the postexposure conditions of the present experiment: The apparent movement of the cue toward the center of the visual field is the major cause of the cue-alignment problems.

\section{GENERAL DISCUSSION}

The partial-report bar-probe task introduced by Averbach and Coriell (1961) is a highly complex visual information-processing and selective-attention task. It therefore comes as no surprise that it takes some time before it reveals some of its major secrets. It is, however, quite amazing, and even somewhat disturbing, to see how long it takes before it is known what the relevant data that such a complex task produces are. In the 35-yearlong history of the bar-probe task, three stages in the process of discovering these data can be distinguished.

Initially, following Averbach and Coriell (1961), only percentages correct reports as a function of spatial position and of moment of presentation of the cue were regarded as pertinent (see van der Heijden, 1992, chap. 5, for an overview). These percentages correct reports were taken as unbiased measures of the subject's ability to identify the cued items. If the subject made an error, it was taken for granted that the error was a failure of perceptual analysis or of identification (see, e.g., Estes, 1978).

More than 10 years later, Mewhort and colleagues made clear that a detailed analysis of the errors is of crucial importance. They discovered that most errors were location 
errors and that most location errors were near-location errors (see, e.g., Mewhort et al., 1981; see van der Heijden, 1992, chap. 5, for an overview). This finding strongly indicated that problems of location, rather than problems of perceptual analysis or identification, impose major limitations on performance in partial-report bar-probe tasks (see, e.g., Campbell \& Mewhort, 1980; Mewhort, 1987; Mewhort et al., 1981; Mewhort et al., 1982).

Now, again more than 10 years later, it appears that the great majority of the near-location errors consist of central near-location errors (i.e., of names of letters that were exposed in central positions relative to the position of the cue) and, furthermore, that an abundance of (near-) location errors is observed not only with postexposure of the cue but also with preexposure. These new findings support Mewhort and colleagues' view on the importance of the location process. Moreover, these observations strongly suggest that location problems arise mainly because, for one or another reason, the cue is perceived as displaced in the direction of the center of the visual field.

\section{Generality}

When new findings are obtained with an old task, critical questions arise. The first question concerns the generality: Are the new findings unique for the present experiments, or is it reasonable to assume that they also could have been observed in the experiments reported earlier in the literature? For at least four reasons, we are quite convinced that our findings are not unique.

First, in all partial-report bar-probe experiments performed in our laboratory, we attempted to stay as close as possible to the method of experimentation described in the literature, especially to the experimental conditions used by Mewhort and colleagues (for descriptions of these attempts, see, e.g., Hagenaar \& van der Heijden, 1995).

Second, in correspondence with these attempts, the postcue condition in our letter-naming experiment (Experiment 2) produced the usual outcomes for all dependent measures (i.e., the results that are generally reported in the literature; for details, see the Discussion section of Experiment 2).

Third, our digit-naming experiment (Experiment 1) yielded, for the conventional dependent measures, a pattern of results that is in all respects comparable to that in Mewhort and Campbell's (1978) digit-naming task.

And fourth, in the only set of data from another laboratory that we were able to analyze - the data reported by Mewhort et al. (1981) - a similar trend is observed for the critical dependent measure (i.e., the direction of the location errors; see the data in Table 3 ).

Taken together, it seems that there is not much reason to assume that the phenomenon of central near-location errors observed in our experiments could not have been observed in the experiments reported earlier in the literature. It is much more likely that the phenomenon was simply not noted.

\section{Relative Importance of Cue Mislocation}

The second question is the question of the relative importance of central-location errors that are induced by apparent cue displacement in regular partial-report barprobe tasks. It is well known that, in these tasks, various error sources contribute to the total pattern of results. For answering the above question, it is worthwhile to elaborate the cue-displacement hypothesis in more detail, specifying the assumptions that underlie it.

The total number of location errors observed on the trials with the cue pointing at target position $t, L_{t}$, can be represented by

$$
L_{t}=C_{t}+P_{t}+O_{t},
$$

where $C_{t}$ is the number of times that the cue was perceived as pointing at a more central position and the item at that position was correctly named, $P_{t}$ is the number of times that the cue was perceived as pointing at a more peripheral position and the item in that position was correctly named, and $O_{t}$ is the number of times that other problems underlay a location error (e.g., identification problems due to: acuity problems, lateral masking, response bias, etc.).

Because many factors contribute to the location errors collected in $O_{t}$, it is reasonable to assume that, for all positions together, the location errors in $O_{t}$ are symmetrically distributed around the indicated position. Then, summed over target positions, the number of central location errors, $\Sigma L_{t}^{c}$, equals $\left(\sum C_{t}+1 / 2 \sum O_{t}\right)$ and the number of peripheral location errors, $\sum L_{t}^{p}$, equals $\left(\sum P_{t}+\right.$ $\left.1 / 2 \Sigma O_{t}\right)$. This allows us to define a parameter, $R I$, by

$$
\begin{aligned}
R I & =\frac{\sum L_{t}^{c}-\sum L_{t}^{p}}{\sum L_{t}} \\
& =\frac{\left(\sum C_{t}+\frac{1}{2} \sum O_{t}\right)-\left(\sum P_{t}+\frac{1}{2} \sum O_{t}\right)}{\sum C_{t}+\sum P_{t}+\sum O_{t}} \\
& =\frac{\sum C_{t}-\sum P_{t}}{\sum C_{t}+\sum P_{t}+\sum O_{t}} .
\end{aligned}
$$

This parameter $R I$ can be regarded as an unbiased estimate of the extent of asymmetry in, or the extent of skewness of, the distributions of location errors around the target positions, expressed as a proportion of the total number of location errors.

Rauk and Luuk's (1980) finding that the cue appears to have shifted toward the fixation point entails that $C_{t}>P_{t}$. Therefore, the hypothesis we derived from Rauk and Luuk's observation for bar-probe experiments implies that $R I$ must be greater than zero. Calculation of $R I$ shows that, in regular bar-probe tasks, .49 (Hagenaar \& van der Heijden, 1995), .30 (Mewhort et al., 1981), and .54 (the postcue condition of Experiment 2) of the nearlocation errors can unambiguously be ascribed to subjects' inclination to perceive the cue as having shifted in 
the direction of the fixation point. ${ }^{1}$ The values are substantial, and they unmistakably indicate the importance of this factor. These values, however, are also evidence that, in regular bar-probe tasks, further error sources must substantially contribute to the overall number of (near-)location errors.

The digit-naming task of Experiment 1 can yield some insight into the origin of some of these errors. For that task, $R I$ equals .84 , indicating that .84 of the location errors can unequivocally be ascribed to an apparent central displacement of the cue and .16 to other sources. In the digit-naming task, with a high value of $R I$, identification problems are very unlikely, due to the use of the predictable array. In the letter-naming tasks, with intermediate values of $R I$, identification failures often occur. This pattern of results suggests that, in the letter-naming task, identification difficulties may prevent the appearance of central location errors.

If this reasoning is correct, we should find, in Experiment 2, a higher $R I$ value for the precuing condition, with impending clear letter-identity information, than for the postcuing condition, with already fading identity information. The values obtained were .71 and .54 for the precuing and the postcuing conditions, respectively. In sum, it seems that apparent cue displacements form a major source of location errors in regular partial-report bar-probe tasks but that identification problems can obscure the importance of this source.

\section{Locating and Identifying}

A third question, which now arises, is about the effect of misidentifications on the error pattern. As stated, the partial-report bar-probe task is a highly complex visual information-processing and selective-attention task. However, with a simple numerical example, we can sketch in broad lines how identification errors can obscure the importance of apparent central cue displacements in a regular partial-report bar-probe task. In this numerical example, we look at what happens when the barmarker indicates Position 3. For this position, we assume that, on .75 of the trials, the cue is correctly located. For the remaining . 25 of the trials, we assume that, in .90 of the cases, the more central Position 4 is seen as the indicated position, and, in 10 of the cases, the more peripheral Position 2 is seen as the indicated position. With these parameters and without misidentification, for this Position 3 , the value of parameter $R I$ equals $.25 \times[(.90-$ $.10) /(.90+.10)]=(.225-.025) /(.225+.025)=.80, \mathrm{a}$ value close to the overall value of .84 obtained for the digit-naming task of Experiment 1.

With regard to identification, we assume that the probability of a correct identification is .50 (see van der Heijden, 1992, chap. VI, for estimates for inner positions of .60 and .40$)$. In addition, we assume that, given an erroneous identification, all remaining 25 letters of the alphabet have a probability of .04 of being named. It is now easy to see how identification problems can conceal the significance of apparent central cue displacements. Two different factors are involved.
First, with correct location but incorrect identification, $.0150(=.75 \times .50 \times .04)$ of the responses show up as central near-location errors and .0150 as peripheral near-location errors. Because this factor contributes equally to the two error types, it obscures the importance of central cue displacements.

Second, with incorrect location, $.1125(=.25 \times .90 \times$ $.50)$ of the responses show up as central near-location errors and $.0045(=.25 \times .90 \times .50 \times .04)$ as peripheral near-location errors when the cue is seen at the more central Position 4, and $.0125(=.25 \times .10 \times .50)$ as peripheral near-location errors and $.0005(=.25 \times .10 \times$ $.50 \times .04)$ as central near-location errors when the cue is seen at the more peripheral Position 2. Because, with centrally biased cue perception, more responses show up as peripheral near-location errors (.0045) than as central near-location errors with peripherally biased cue perception (.0005), this factor conceals the significance of central cue displacements.

With these parameters for this position, the total proportion of central near-location errors equals $.0150+$ $.1125+.0005=.1280$, and the total proportion of peripheral near-location errors equals $.0150+.0045+$ $.0125=.0320$. The value of parameter $R I$ equals $(.1280$ $-.0320) /(.1280+.0320)=.60$. This value is considerably smaller than the value of .80 without identification problems and is rather close to the overall values of .71 and .54 obtained for the precuing and postcuing conditions of Experiment 2 (see also Table 4).

\section{Related Research}

A fourth, and last, question concerns the compatability of the main finding - cue alignment problems are the major source of location errors in regular partial-report bar-probe tasks - with other results reported in the literature. Of course, we are not able to evaluate all related research here. We therefore restrict our discussion to the type of task used in the present study: tasks in which position of the relevant information is visually indicated, and subjects have to respond with identity. Five groups of results that, at first sight, are not compatible with our results are worth mentioning.

First, with circular arrays, in which peripheral to central movements of the cue would be irrelevant, an increasing number of location errors with increasing cue delay is found (see, e.g., Dixon \& Di Lollo, 1991; Eriksen \& Rohrbaugh, 1970; Hagenaar \& van der Heijden, 1995). The numerical example just presented explains this result. With correct location but incorrect identification, a proportion of the misidentifications shows up as location errors. Because, with increasing cue delays, the number of misidentifications increases, the number of location errors also increases. (In this context, it is worthwhile to note that, in line with our cue-displacement hypothesis, under comparable conditions, the number of location errors is much smaller with circular arrays than with linear arrays; see Hagenaar \& van der Heijden, 1995.)

Second, there is evidence suggesting that, with circular arrays and increasing preexposure of the cue, a de- 
creasing number of location errors is obtained (see, e.g., Eriksen \& Rohrbaugh, 1970). As already stated, however, with circular arrays, any peripheral-to-central movement of the cue is irrelevant; the cue still indicates the same spatial position. An effect of apparent movement of the cue toward the point of fixation as observed in Experiment 2 is to be expected only with linear arrays, as used in our experiments.

Third, there is evidence showing that location errors are also more frequent than other types of errors when subjects report the position of the cue accurately (see, e.g., Irwin, 1992). An explanation for this phenomenon in terms of identification problems and subjects' report strategies was provided by van der Heijden (1986, p. 217). Assume that, in a subject's experience, the cue does not point to a single letter position but to an area containing a small group of letter positions, with different probabilities of being indicated for the individual positions. For instance, assume that, on a trial, the cue points with a (subjective) probability of .75 to the correct position and of .25 to a neighboring position. Assume further that, for this subject, the probability of correctly naming the letter in the target position is .20 , and the probability of correctly naming the letter in the neighboring position is .90 . Then, for this subject, the probability of giving a correct response equals $.75 \times .20=.15$ for the target position and $.25 \times .90=.23$ for the adjacent position. What this example illustrates is that, when identification is difficult, naming a letter adjacent to the (what is perceived as the most likely) target position can be the optimum strategy.

Fourth, there is abundant evidence that locating of items is also imperfect when, instead of a spatial cue such as a barmarker, another type of cue (e.g., the color of an item) is used to indicate the position of the item(s) to be named (see, e.g., Butler, Mewhort, \& Tramer, 1987; Fryklund, 1975; Snyder, 1972; van der Heijden, 1993). An explanation for this result was provided by van der Heijden (1993, p. 52). Basically, this explanation states that a color, just as a barmarker or an arrow, performs its function by indicating through its position the position of the relevant element. Whether with colors the same pattern of location errors is observed as in the experiments here reported is, of course, a matter for further investigation.

Fifth, and lastly, it has been reported that location errors are also more frequent than other types of errors when an entire row rather than a single letter has to be reported (see, e.g., Irwin \& Brown, 1987; Irwin \& Yeomans, 1986). This brings us back to the introduction of this paper. When, in a partial-report task, an entire row has to be named, the task turns into a kind of task fairly similar to Estes et al.'s (1976) whole-report task. Estes observed that, in this task, the subjects made many "transposition errors." In our Discussion section of Experiment 1, we stated that our partial-report bar-probe results do not provide any support for such an apparent displacement of items. It is therefore highly likely that these whole-report errors occur at output rather than during perception. In other words, it is very probable that these observations are irrelevant for the issue we are concerned with. Taken together, it seems that there is little reason to assume that the pattern of results here reported is incompatible with the available experimental evidence.

\section{Cue Displacement}

Fortunately, the cue-displacement hypothesis is not just an ad hoc hypothesis only invoked to explain an interesting feature of the data obtained in partial-report bar-probe tasks. Outside the field of visual information processing and selective attention, evidence for the apparent displacement of an object in the direction of the point of fixation has been reported more than once. In the introduction of this paper, we described Rauk and Luuk's (1980) research, which indicated a general tendency to estimate the position of an object more centrally than it actually is. Similar or closely related findings have been reported by Bedell and Flom (1981), Leibowitz, Myers, and Grant (1955), Mateeff and Gourevich (1983, 1984), Mateeff and Hohnsbein (1988), Mitrani and Dimitrov (1982), O'Regan (1984), Osaka (1977), Rose and Halpern (1992), and Skavenski (1990). So, it appears that we can use a well-established fact for the explanation of a newly detected phenomenon: the central near-location errors.

Unfortunately, the literature just referred to does not give a clear answer to the question of under what conditions an object appears $t n$ be displaced in the direction of the point of fixation. There is, however, a feature in our data that possibly bears some relevance to this matter. The function relating proportions of near-location errors to SOAs in Figure 2a shows that this proportion is smallest when the cue immediately follows the array $(\mathrm{SOA}=$ $50 \mathrm{msec}$ ) and increases with increasing pre- and postexposure intervals. The same dependence on SOA emerges if only the central near-location errors for targets on Positions $2,3,5$, and 6 are considered: .249, .256, .244, .159, $.140, .182$, and .221 for SOAs from -150 to $150 \mathrm{msec}$, respectively. This pattern of results suggests that, especially with a temporal separation between cue and array, apparent displacements of the cue in the direction of the center of the visual field are observed. In other words, the array may be of some help in accurately fixing the true position of the cue. But, of course, this issue deserves much more investigation.

Neither the literature nor our experiments provide unambiguous clues as to why an object is seen as displaced. Further work with position-judgment tasks and with partial-report bar-probe tasks will be necessary to answer this and related questions. Our present research is concerned with the issue.

\section{REFERENCES}

Averbach, E., \& Coriell, A. S. (1961). Short-term memory in vision Bell System Technical Journal, 40, 309-328

BedEll, H. E., \& Flom, M. C. (1981). Monocular spatial distortion in strabismic amblyopia. Investigative Ophthalmology \& Visual Science, 20, 263-268.

Butler, B. E., Mewhort, D. J. K., \& Tramer, S. C. (1987). Location 
errors in tachistoscopic recognition: Guesses, probe errors, or spatial confusion? Canadian Journal of Psychology, 41, 339-350.

Campbell, A. J., \& Mewhort, D. J. K. (1980). On familiarity effects in visual information processing. Canadian Journal of Psychology, 34, 134-154.

Dixon, P., \& Di Lollo, V. (1991). Effects of display luminance, stimulus type, and probe duration on visible and schematic perception. Canadian Journal of Psychology, 45, 54-74.

ERIKSEN, C. W. (1990). Attentional search of the visual field. In D. Brogan (Ed.), Visual search (pp. 3-19). London: Taylor \& Francis.

ERIKSEN, C. W., \& Rohrbaugh, J. W. (1970). Some factors determining efficiency of selective attention. American Journal of Psychology, 83, 330-342.

Estes, W. K. (1978). Perceptual processing in letter recognition and reading. In E. C. Carterette \& M. P. Friedman (Eds.), Handbook of perception $I X$ (pp. 163-220). New York: Academic Press.

Estes, W. K., Allmeyer, D. H., \& Reder, R. M. (1976). Serial position functions for letter identification at brief and extended exposure durations. Perception \& Psychophysics, 19, 1-15.

FRYKLUND, I. (1975). Effects of cued-set spatial arrangement and target-background similarity in the partial-report paradigm. Perception \& Psychophysics, 17, 375-386.

HagenaAR, R., \& van Der HeIJden, A. H. C. (1995). On the relation between type of arrays and type of errors in partial-report bar-probe studies. Acta Psychologica, 88, 89-104.

Hagenzieker, M. P., van der HeiJden, A. H. C., \& HagenaAR, R. (1990). Time courses in visual-information processing: Some empirical evidence for inhibition. Psychological Research, 52, 13-21.

HaRCUM, E. R. (1964). An isolation effect in pattern perception similar to that in serial learning. Perceptual \& Motor Skills, 19, 855-860.

Harcum, E. R. (1970). Defining shape for perceptual element-position curves. Psychological Bulletin, 74, 362-372.

IRWIN, D. E. (1992). Memory for position and identity across eye movements. Journal of Experimental Psychology: Learning, Memory, \& Cognition, 18, 307-317.

IRWIN, D. E., \& BRown, J. S. (1987). Tests of a model of informational persistence. Canadian Journal of Psychology, 41, 317-338.

Irwin, D. E., \& Yeomans, J. M. (1986). Sensory registration and informational persistence. Journal of Experimental Psychology: Human Perception \& Performance, 12, 343-360.

KIRK, R. E. (1968). Experimental design: Procedures for the behavioural sciences. Belmont, CA: Brooks/Cole.

KRUMHANSL, C. L. (1977). Naming and locating simultaneously and sequentially presented letters. Perception \& Psychophysics, 22, 293302.

Krumhansl, C. L., \& Thomas, E. A. C. (1976). Extracting identity and location information from briefly presented letter arrays. Perception \& Psychophysics, 20, 243-258.

KrumhansL, C. L., \& Thomas, E. A. C. (1977). Effect of level of confusability on reporting letters from briefly presented visual displays. Perception \& Psychophysics, 21, 269-279.

Leibowitz, H. W., Myers, N. A., \& Grant, D. A. (1955). Frequency of seeing and radial localization of single and multiple visual stimuli. Journal of Experimental Psychology, 50, 369-373.

LOWE, D. G. (1975). Processing of information about location in brief visual displays. Perception \& Psychophysics, 18, 309-316.

Mateeff, S., \& Gourevich, A. (1983). Peripheral vision and perceived visual direction. Biological Cybernetics, 49, 111-118.

MateefF, S., \& Gourevich, A. (1984). Brief stimuli localization in visual periphery. Acta Physiologica et Pharmacologica Bulgarica, 10, 64-71.
MateefF, S., \& Hohnsbein, J. (1988). Perceptual latencies are shorter for motion towards the fovea than for motion away. Vision Research, $28,711-719$

MEwhoRT, D. J. K. (1987). Information stores and mechanisms: Early stages in visual processing. In H. Heuer \& A. F. Sanders (Eds.), Perspectives on perception and action (pp. 335-357). New York: Erlbaum.

Mewhort, D. J. K., Butler, B. E., Feldman-Stewart, D., \& Tramer, S. (1988). "Iconic memory," location information, and the bar-probe task: A reply to Chow (1986). Journal of Experimental Psychology: Human Perception \& Performance, 14, 729-737.

Mewhort, D. J. K., \& Camprell, A. J. (1978). Processing spatial information and the selective-masking effect. Perception \& Psychophysics, 24, 93-101.

Mewhort, D. J. K., Campeell, A. J., MarChetti, F. M., \& Campbell J. I. D. (1981). Identification, localization, and "iconic memory": An evaluation of the bar-probe task. Memory \& Cognition, 9, 50-67.

Mewhort, D. J. K., Marchetti, F. M., \& Campbell, A. J. (1982). Blank characters in tachistoscopic recognition: Space has both a symbolic and a sensory role. Canadian Journal of Psychology, 36, 559-575.

Mitrani, L., \& Dimitrov, G. (1982). Retinal location and visual localization during pursuit eye movement. Vision Research, 22, 1047 1051

O'REGAN, J. K. (1984). Retinal versus extraretinal influences in flash localization during saccadic eye movements in the presence of a visible background. Perception \& Psychophysics, 36, 1-14.

OSAKA, N. (1977). Effect of refraction on perceived locus of a target in the peripheral visual field. Journal of Psychology, 95, 59-62.

RaUk, M., \& LUUK, A. (1980). Identification and detection of spatial position in one-dimensional pattern. In Problems of cognitive psychology (Acta et Commentationes Universitatis Tartuensis, Vol. 522 , pp. 143-163). Tartu, Estonia: Tartu Riiklik Ülikool.

Rose, D., \& HALPERN, D. L. (1992). Stimulus mislocalization depends on spatial frequency. Perception, 21, 289-296.

SKAVENSKI, A. A. (1990). Eye movement and visual localization of objects in space. In E. Kowler (Ed.), Eye movements and their role in visual and cognitive processes (pp. 263-287). Amsterdam: Elsevier.

SNYDER, C. R. R. (1972). Selection, inspection and naming in visual search. Journal of Experimental Psychology, 92, 428-431

TOWNSEND, V. M. (1973). Loss of spatial information following a tachistoscopic exposure. Journal of Experimental Psychology, $\mathbf{9 8}$ 113-118.

van Der Hejden, A. H. C. (1986). On selection in vision. Psychological Research, 48, 211-219.

VAN DER HEIJden, A. H. C. (1992). Selective attention in vision. New York: Routledge.

van DER Heijden, A. H. C. (1993). The role of position in object selection in vision. Psychological Research, 56, 44-58.

\section{NOTE}

1. To equalize in our calculations the number of positions that can deliver erroneous response letters, only positions, adjacent to the target and only target positions with at least one position on either side in the same visual half field were taken into account (i.e., Target Positions 2, 3, 5, and 6 for seven-item arrays, and Target Positions 2, 3,6, and 7 for eight-letter arrays)

(Manuscript received December 1, 1995; revision accepted for publication July 18, 1996.) 